Бабаев А.Б., Муртазаев А.К., Атаева Г.Я. Термодинамические и критические свойства сильно разбавленной низкоразмерной антиферромагнитной модели Поттса на треугольной решетке

УДК 537.9

DOI: $10.21779 / 2542-0321-2018-33-2-40-44$

А.Б. Бабаев ${ }^{1,2}$, А.К. Муртазаев ${ }^{1,3}$, Г.Я. Атаева ${ }^{1}$

Термодинамические и критические свойства сильно разбавленной низкоразмерной антиферромагнитной модели Поттса на треугольной решетке

${ }^{1}$ Институт физики им. Х.И. Амирханова Дагестанского научного иентра РАН; Россия, 367003, г. Махачкала, ул. М. Ярагского, 94; b_albert78@mail.ru;

2 Дагестанский научный иентр РАН; Россия, 367025, г. Махачкала, ул. М. Гаджсиева, 45;

3 Дагестанский государственный университет; Россия, 367001, Махачкала, ул. М. Гаджиева, $43 a$

Методом Монте-Карло исследованы термодинамические и критические свойства сильно разбавленной низкоразмерной антиферромагнитной модели Поттса на треугольной решетке. Расчеты проводились для систем с периодическими граничными условиями. Исследовались системы с линейными размерами $L \times L=N, L=20-120$. Построены зависимости термодинамических параметров от температуры. На основе метода кумулянтов Биндера четвертого порядка показано, что внесение вмороженных немагнитных примесей в чистую систему индуцирует фазовый переход второго рода. Этим методом вычислены температуры фазовых переходов для всех рассмотренных значений $p$. Определены статические критические индексы восприимчивости $\gamma$, намагниченности $\beta$, теплоемкости $\alpha$ и индекс радиуса корреляции $v$ для рассмотренной модели при концентрации спинов $p=0.70,0.65$. Показано, что вычисленные критические индексы в сильно разбавленном режиме в пределах погрешности удовлетворяют соотношениям теории конечно-размерного скейлинга.

Ключевые слова: критические индексы, кумулянты Биндера, примеси, фазовые переходы, вмороженный беспорядок, универсальность, модель Поттса.

Методы Монте-Карло (МК) оказались востребованными для исследования критических свойств магнитных систем с немагнитными примесями. Это связано с тем, что неидеальные черты, являющиеся неотъемлемой частью реальных твердых тел, оказывают значительное влияние на фазовые переходы (ФП) и критические явления (КЯ) магнитных систем. Существующие же теоретические методы перестают работать в сильно разбавленном режиме, а результаты экспериментальных исследований сильно зависят не только от метода и конкретного образца, но и от способа приготовления образца [1].

Применение методов МК позволило изучать более реалистичные модели с присущими им примесями и дефектами структур, учитывать усложняющие факторы, всегда присутствующие в реальных материалах [1-3]. 
Бабаев А.Б., Муртазаев А.К., Атаева Г.Я. Термодинамические и критические свойства сильно разбавленной низкоразмерной антиферромагнитной модели Поттса на треугольной решетке

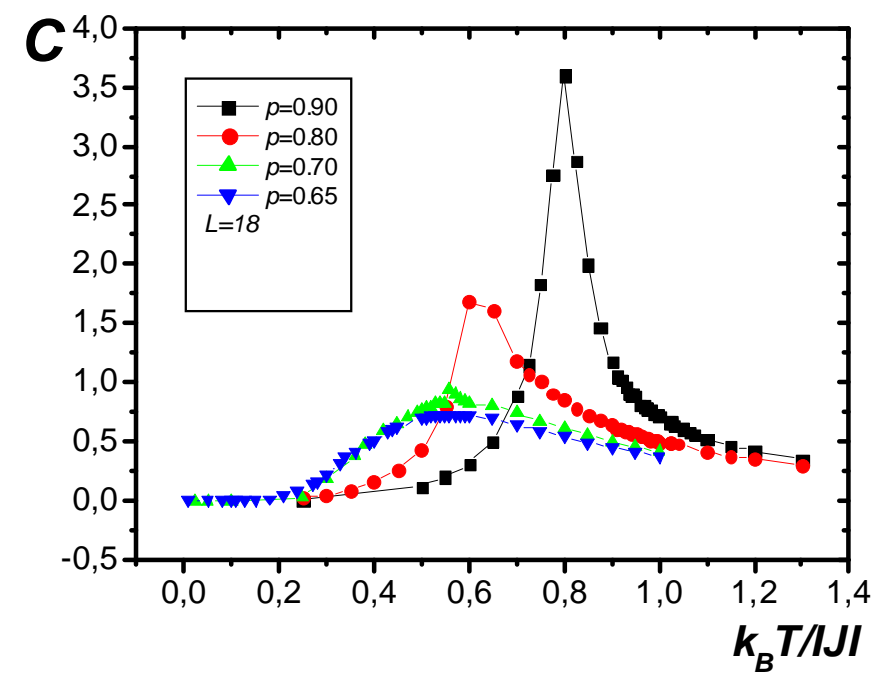

Рис. 1. Температурная зависимость теплоемкости $C$ для сильно разбавленной АФ модели Поттca
Основной целью данной работы является исследование термодинамических свойств и расчет критических параметров сильно неупорядоченных систем, описываемых антиферромагнитной (АФ) моделью Поттса на треугольной решетке при концентрации спинов $p=0.65$ на основе методов Монте-Карло. Интерес к АФ модели Поттса на треугольной решетке обусловлен тем, что только на этой решетке наблюдается фазовый переход. Кроме того, многие физические объекты и явления описываются этой моделью на треугольной решетке [4].

Рассматривались системы с периодическими граничными условиями (ПГУ) и линейными размерами $L \times L=N, L=9-144$. При построении сильно разбавленной модели Поттса следует учесть следующие особенности: в узлах треугольной решетки расположены спины $S_{i}$, которые могут находиться в одном из $q>2$ состояний, и немагнитные примеси; немагнитные примеси расположены случайно и фиксированы в узлах решетки.

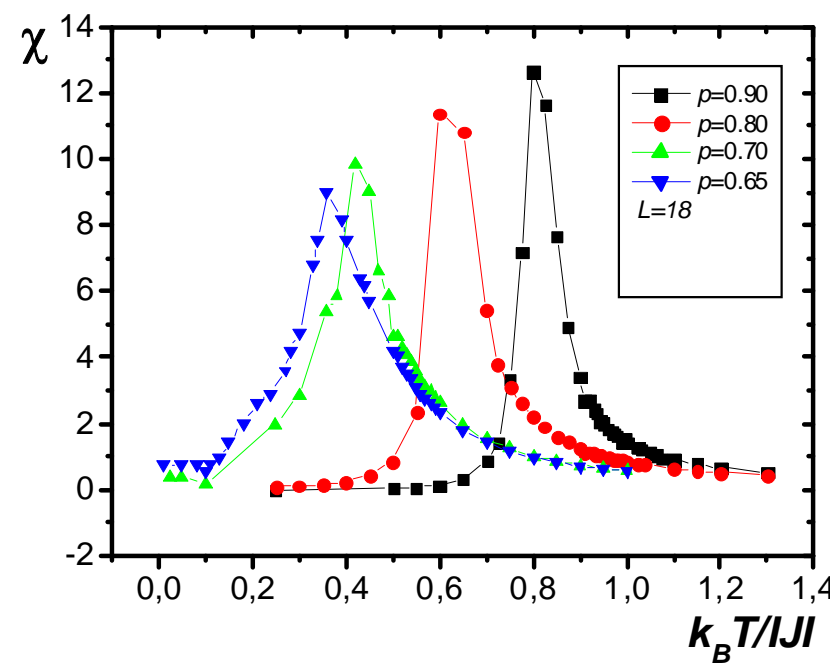

Рис. 2. Температурная зависимость восприимчивости $\chi$ для сильно разбавленной АФ модели Поттса на треугольной решетке.
Гамильтониан рассматриваемой системы имеет вид [5].

$$
H=-\frac{1}{2} J \sum_{i, j} \rho_{i} \rho_{j} \cos \theta_{\mathrm{i}, \mathrm{j}}, \quad S_{i}=1,2,3,
$$

где $J$ - параметр обменного АФ взаимодействия ближайших соседей $(J<0)$; $\rho_{i}=1$, если узел $i$ занят магнитным атомом, $\rho_{i}=0$, если в узле находится немагнитная примесь; $\theta_{i, j}-$ угол между взаимодействующими спинами $S_{i}-S_{j}$.

В работе [6] на основе метода кумулянтов Биндера $[7,8]$ было показано, что в сильно разбавленной модели Поттса при $p=0.65$ и $T=T_{c}=0.35$ (4) наблюдается ФП второго рода. Методика определения рода ФП этим методом подробно описана в работах [9-13].

На рис. 1-3 представлены характерные зависимости теплоемкости $C$, восприимчивости $\chi$ и параметра порядка $m_{A F}$ от температуры $T$ при концентрации спинов $p=0.90,0.80$, $0.70,0.65$. Здесь и далее погрешность данных не превышает размеры использованных символов на рисунках. Как видно из рис. 1 и 2, наличие немагнитных примесей приво- 
Бабаев А.Б., Муртазаев А.К., Атаева Г.Я. Термодинамические и критические свойства сильно разбавленной низкоразмерной антиферромагнитной модели Поттса на треугольной решетке

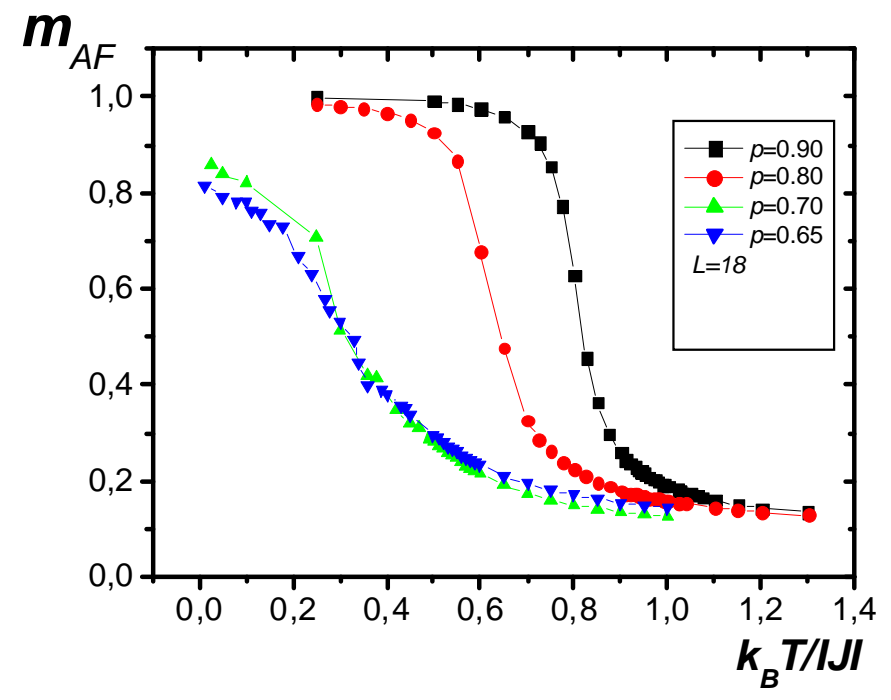

Рис. 3. Температурная зависимость параметра порядка $m_{A F}$ для сильно разбавленной АФ модели Поттса на треугольной решетке дит к сглаживанию максимумов теплоемкости и восприимчивости, а также к их уменьшению с ростом концентрации примесей.

Для всех рассмотренных систем, в которых наблюдается ФП второго рода, нами на основе теории конечноразмерного скейлинга (КРС) рассчитывались статические критические индексы (КИ): теплоемкости $\alpha$, восприимчивости $\gamma$ и параметра порядка $\beta$. Из соотношений этой теории следует, что для достаточно большой системы с ПГУ при температуре $T=T c$, параметр порядка $m_{A F}$, восприимчивость $\chi$ и

параметр $V_{n}$ для определения критического индекса $v$ удовлетворяют следующим аналитическим выражениям $[14,15]$ :

$$
\begin{aligned}
& m_{A F} \sim L^{-\beta / v}, \\
& \chi \sim L^{\gamma / v}, \\
& V_{n}=L^{1 / v} g_{V_{n}},
\end{aligned}
$$

где $g_{V n}-$ некоторая постоянная, а в качестве $V_{n}$ могут выступать:

$$
\begin{gathered}
V_{n}=\frac{\left\langle m_{A F}{ }^{n} E\right\rangle}{\left\langle m_{A F}{ }^{n}\right\rangle}-\langle E\rangle,(n=1,2,3), \\
V_{3}=\frac{d U_{L}}{d \beta}=\frac{1}{3\left\langle m_{A F}{ }^{2}\right\rangle^{2}}\left[\left\langle m_{A F}^{4}\right\rangle\langle E\rangle-2 \frac{\left\langle m_{A F}{ }^{4}\right\rangle\left\langle m_{A F}{ }^{2} E\right\rangle}{\left\langle m_{A F}{ }^{2}\right\rangle^{2}}+\left\langle m_{A F}^{4} E\right\rangle\right],
\end{gathered}
$$

где $\beta=1 / T, T-$ температура.

Для аппроксимации температурной зависимости теплоемкости от $L$, как правило, используются другие выражения, например [8]:

$$
C_{\text {max }}(L)=C_{\text {max }}(L=\infty)-A L^{\alpha / v},
$$

где $A$ - некоторый коэффициент.

Далее для расчета КИ $\alpha, \beta, \gamma$ и $v$ строились зависимости $C, m_{A F}, \chi$, и $V_{n}$ от $L$ в двойном логарифмическом масштабе при $T=T_{C}$ и $p=0.70,0.65$. Анализ данных, выполненный с использованием нелинейного метода наименьших квадратов, позволил определить значения $\frac{\alpha}{v}=0.362(3), \frac{\beta}{v}=0.146(2), \frac{\gamma}{v}=1.647(3) \quad$ и $\quad \frac{1}{v}=0.98$ (3) при $p=0.65$. Затем с использованием значения $v=1.02(2)$, полученного в рамках данного исследования, были определены следующие индексы: $\alpha=0.369(3), \beta=0.148$ (2), 
Бабаев А.Б., Муртазаев А.К., Атаева Г.Я. Термодинамические и критические свойства сильно разбавленной низкоразмерной антиферромагнитной модели Поттса на треугольной решетке

$\gamma=1.685(3), v=1.02(1)$. Полученные критические индексы в пределах погрешности удовлетворяют соотношению теории КРС $d v=\gamma+2 \beta$. Более подробно методика определения КИ с использованием теории КРС приведена в работе [16].

Таким образом, в настоящей работе на основе теории КРС рассчитаны статические КИ: теплоемкости $\alpha$, восприимчивости $\gamma$, параметра порядка $\beta$ и индекс радиуса корреляции $v$ для двумерной сильно разбавленной АФ модели Поттса с $q=3$ на треугольной решетке при $p=0.65$. Рассчитанные значения отношений КИ $\frac{\alpha}{v}, \frac{\beta}{v}, \frac{\gamma}{v}$ и $\frac{1}{v}$ удовлетворяют соотношениям теории КРС $\frac{2 \beta}{v}+\frac{\gamma}{v}=d$.

Исследование выполнено при финансовой поддержке РФФИ в рамках научного проекта №16-02-00214-а.

\section{Литература}

1. Фольк Р., Головач Ю., Яворский Т. Критические показатели трехмерной слабо разбавленной замороженной модели Изинга // УФН. - 2003. - Т. 173. - С. 175-200.

2. Доценко В.С. Критические явления в спиновых системах с беспорядком // УФН. - 1995. - Т. 165, № 5. - С. 481-528.

3. Муртазаев А.К., Камилов И.К., Бабаев А.Б. Критическое поведение трехмерной модели Изинга с вмороженным беспорядком на кубической решетке // ЖЭТФ. - 2004. T. 126, № 6. - С. 1377-1383.

4. Муртазаев А.К., Бабаев А.Б., Магомедов М.А., Кассан-Огль Ф.А., Прошкин А.И. Фрустрации и фазовые переходы в трехвершинной модели Поттса на треугольной решетке с взаимодействиями вторых ближайших соседей // Письма в ЖЭТФ. - 2014. T. 100, № 4. - C. 267-271.

5. Wu F.Y. The Potts model // Rev. Mod. Phys. - 1982. - V. 54. - P. 235-268.

6. Murtazaev A.K., Babaev A.B., Ataeva G.Y. Investigation of the thermodynamic properties and phase transitions in a strongly diluted three-vertex antiferromagnetic Potts model by the Monte Carlo method // Physics of Solid State. - 2017. - V. 59. - P. 141-144.

7. Eichhorn K., Binder K. Monte Carlo investigation of the three-dimensional randomfield three-state Potts model // J. Phys.: Cond. Matter. - 1996. - V. 8. - P. 5209-5227.

8. Loison D., Schotte K.D. First and second order transition in frustrated XY systems // Eur. Phys. J. B. -1998. - V. 5. - P. 735-743.

9. Murtazaev A.K., Babaev A.B., Aznaurova G.Y. Phase transitions in 3D site-diluted Potts model with spin states $q=4$ // Solid State Phenomena. - 2011. - V. 168-169. - P. 357360.

10. Murtazaev A.K., Babaev A.B., Aznaurova G.Y. Investigation of the critical properties in the 3d site-diluted Potts model // Solid State Phenomena. - 2009. - V. 152-153. - P. 571574.

11. Муртазаев А.К., Бабаев А.Б., Атаева Г.Я. Фазовые переходы в двумерной ферромагнитной модели Поттса при $\mathrm{q}=3$ на треугольной решетке // Физика низких температур. - 2013. - Т. 39, № 2. - С. 194-198.

12. Муртазаев А.К., Бабаев А.Б. Фазовые переходы в двумерной ферро- и антиферромагнитной моделях Поттса на треугольной решетке // Журнал экспериментальной и теоретической физики. - 2012. - Т. 142, № 6. - С. 1189-1195. 
Бабаев А.Б., Муртазаев А.К., Атаева Г.Я. Термодинамические и критические свойства сильно разбавленной низкоразмерной антиферромагнитной модели Поттса на треугольной решетке

13. Муртазаев А.К., Бабаев А.Б. Трикритическая точка трехмерной модели Поттса $(\mathrm{q}=4)$ с вмороженным немагнитным беспорядком // Письма в ЖЭТФ. - 2014. - V. 99, № 9. - C. 618-622.

14. Loison D. Monte Carlo cluster algorithm for ferromagnetic Hamiltonians // Physics Letters. - 1999. - V. 257. - C. 83-87.

15. Fisher M.E., Barber M.N. Scaling theory for finite-size effects in the critical region // Phys. Rev. Lett. - 1972. - № 28. - P. 1516.

16. Муртазаев А.К., Бабаев А.Б., Атаева Г.Я., Ризванова Т.Р., Джамалутдинов M.P. Компьютерное моделирование критического поведения двумерной слабо разбавленной антиферромагнитной модели Поттса на треугольной решетке // Физика твердого тела. - 2018. - V. 60, № 6. - С. 1169-1172.

Поступила в редакциию 15 февраля 2018 г.

UDC 537.9

DOI: $10.21779 / 2542-0321-2018-33-2-40-44$

\title{
Thermodynamic and critical behavior of a strongly diluted low-dimensional antiferromagnetic Potts model on a triangular lattice
}

\author{
A.B. Babaev ${ }^{1,2}$, A.K. Murtazaev ${ }^{1,3}$, G.Ya. Ataeva ${ }^{1}$ \\ ${ }^{1}$ Institute of Physics, Dagestan Scientific Center, Russian Academy of Sciences; Russia, \\ 367003, Makhachkala, M. Yaragskyst., 94; b_albert78@mail.ru; \\ 2 Dagestan Scientific Center of Russian Academy of Sciences; Russia, 367025, \\ Makhachkala, M. Gadzhiev st., 45; \\ ${ }^{3}$ Dagestan State University; Russia, 367001, Makhachkala, M. Gadzhiev st., $43 a$
}

The thermodynamic and critical properties of the highly dilute low-dimensional antiferromagnetic Potts model on a triangular lattice are studied by the Monte Carlo method. Calculations were carried out for systems with periodic boundary conditions. Systems with linear dimensions $L \times L=N$, $L=20-120$ were studied. Dependences of thermodynamic parameters on temperature are constructed. Based on the fourth Binder order cumulant method, it is shown that the introduction of frozen nonmagnetic impurities into a pure system induces a second-order phase transition. This method calculates the phase transition temperatures for all the $p 0.70,0.65$ values considered. Static critical indices of susceptibility $\gamma$, magnetization $\beta$, heat capacity $\alpha$ and index of correlation radius $v$ for the model considered at spins concentration $p=0.70,0.65$ are determined. It is shown that the calculated critical indices in a strongly diluted regime within the error limits satisfy the relations of the theory of finitedimensional scaling.

Keywords: critical exponents, Binder cumulants, impurities, phase transitions, quenched disorder, universality, Potts model.

Received 15 February, 2018 